Enabl i ng Comput at i onal I nt el I i gence for Green I nt er net of Thi ngs: Dat a Dri ven Adapt at i on i $n$ LPWA Net wor ki ng

\begin{tabular}{|l|l|}
\hline 著者 & ZHANG Chaof eng, DONG M anxi ong, OTA Kaor u \\
\hline $\begin{array}{l}\text { j our nal or } \\
\text { publ i cat i on t i t l e }\end{array}$ & I EEE COMPUTATI ONAL I NTELLI GENCE MAGAZI NE \\
\hline vol une & 15 \\
\hline number & 1 \\
\hline page $\mathrm{r}$ ange & $32-43$ \\
\hline year & 2020 \\
\hline URL & ht t p: //hdl . handl e. net /10258/00010325 \\
\hline
\end{tabular}




\title{
Enabling Computational Intelligence for Green Internet of Things: Data-Driven Adaptation in LPWA Networking
}

Chaofeng Zhang, Advanced Institute of Industrial Technology, Tokyo Metropolitan University Public University Corporation, Tokyo, Japan

Mianxiong Dong, Department of Information and Electronic Engineering, Muroran

Institute of Technology, Muroran, Japan

Kaoru Ota, Department of Information and Electronic Engineering, Muroran Institute of Technology, Muroran, Japan

\begin{abstract}
With the exponential expansion of the number of IoT (Internet of Things) devices, many state-of-the-art communication technologies are being developed to use the lower-power but extensively deployed devices. Due to the limits of pure channel characteristics, most protocols cannot allow an IoT network to be simultaneously largescale and energy-efficient, especially in hybrid architectures. However, different from the original intention to pursue faster and broader connectivity, the daily operation of IoT devices only requires stable and low-cost links. Thus, our design goal is to develop a comprehensive solution for intelligent green IoT networking to satisfy the modern requirements through a data-driven mechanism, so that the IoT networks use computational intelligence to realize self-regulation of composition, size minimization, and throughput optimization. To the best of our knowledge, this study is the first to use the green protocols of LoRa and ZigBee to establish an ad hoc network and solve the problem of energy efficiency. First, we propose a unique initialization mechanism that automatically schedules node clustering and throughput optimization. Then, each device executes a procedure to manage its own energy consumption to optimize switching in and out of sleep mode, which relies on AI-controlled service usage habit prediction to learn the future usage trend. Finally, our new theory is corroborated through real-world deployment and numerical comparisons. We believe that our new type of network organization and control system could improve the performance of all green-oriented IoT services and even change human lifestyle habits.
\end{abstract}

Corresponding Author: Mianxiong Dong (Email: mxdong@mmm.muroran-it.ac.jp) 


\section{INTRODUCTION}

The Internet of Things (IoT) helps make the world that people live in smarter than ever. The world residents living in are incessantly, automatically and collaboratively provided with customized urban services. The IoT contains smart devices (e.g., wearable devices and mobile devices), communication technologies (e.g., wireless ad hoc networks (WANETs) and next-generation cellular networks (NGCNs)), computation infrastructure (e.g., cloud computing and edge computing) and other core technologies (identification, sensing and personalization) [1]. In addition to facilitating these common smart services in daily life, IoT devices have appeared in various environments. Such devices create new industry forms, such as smart healthcare, smart grids [2], and the overall concept of smart cities, and influence home appliances [3], food supply chains and industrial automation [4]. In contrast to the pure IoT networks' need for faster speeds and larger bandwidth, these daily services also need stable, low-cost, and energy-efficient links [5]. The green IoT initiatives are motivated by the full deployment and ever-increasing demand for smart services when the power and bandwidth rather than resources are considered as a limitation [6]. Therefore, new energy-efficient procedures (whether involving hardware or software) should be adopted during the design of IoT services to eliminate waste of resources or reduce the impact of the green procedure itself.

To make the IoT 'green', more state-of-the-art technologies are being considered and newly developed to fulfill the demand of these energy-hungry devices [7]. Regarding wireless communications, numerous IoT nodes are intelligently assembled to transfer the data collected during monitoring [8]. A base station (BS) provides various applications to facilitate communications, and it intelligently adjusts power, controls the protocols, schedules activities and allocates resources. These tasks are easily performed by technologies in the D2D domain. However, most of these tasks consume more energy than a simple IoT device can afford. According to a survey of all the wireless communication technologies [9], medium-distance connectivity methods (e.g., 802.15.4) are good choices for meeting the demand for low-rate and lowpower communications [10] usually involved in smart parking and tracking. Furthermore, long-distance machine-type communications (e.g., LoRa and eMTC) are areas of new and ongoing research. They represent effective approaches applied in meters and public facilities.

Nevertheless, if we use medium-distance communication technologies (such as ZigBee) to form a WANET, the actual delay over multiple hops will significantly increase, and throughput will decline [11]; many redundant packets will have to be generated to achieve a higher delivery rate, which will lead to additional energy consumption. On the other hand, the pure low-power wide area (LPWA) protocols could avoid the above multiple relaying problems, but the unlicensed narrow band significantly limits 


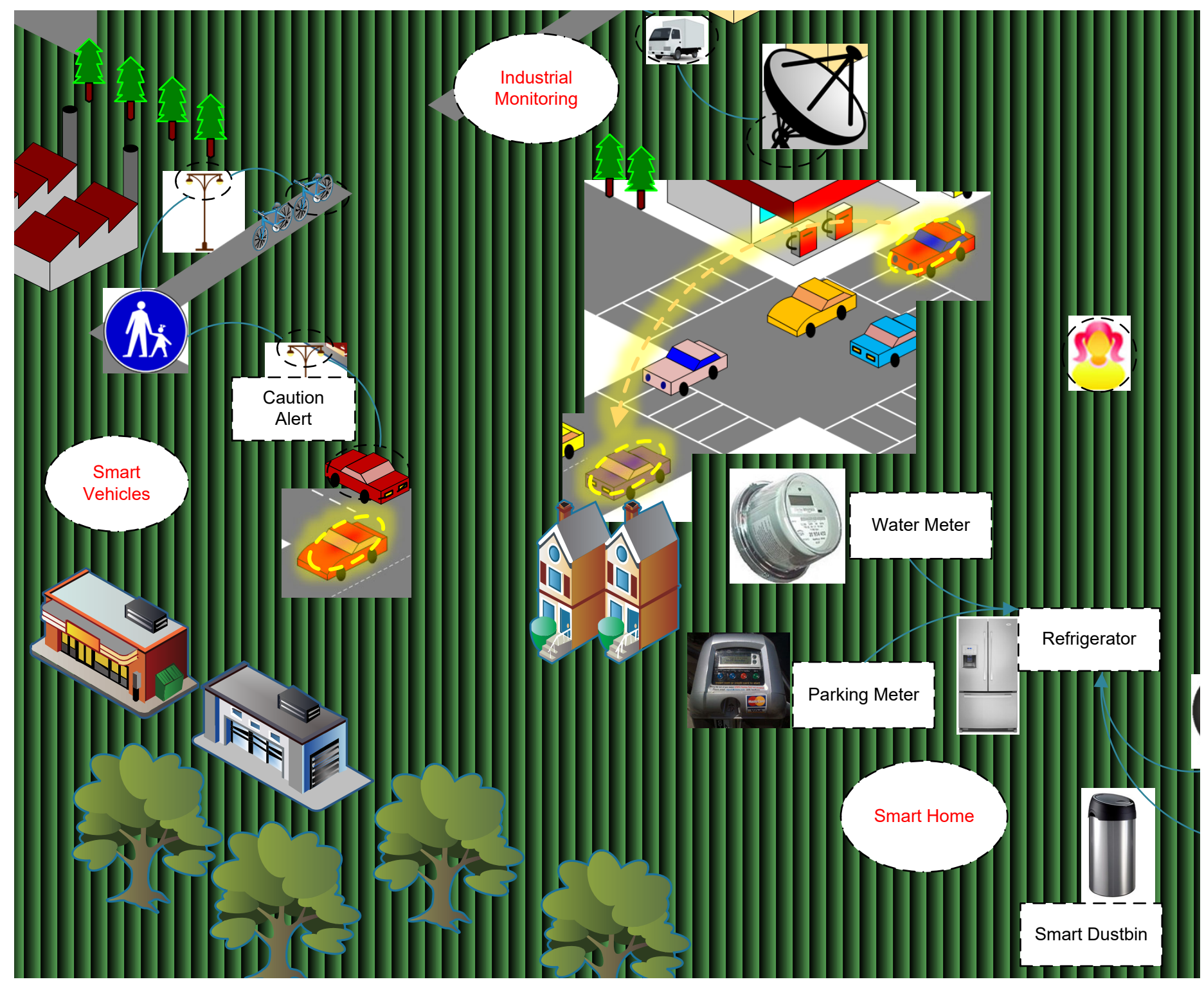

Fig. 1: Simple and low-power yet still useful IoT devices are widely deployed presently.

the performance of multinode networks [12]. In addition to the throughput limitation of the node-to-BS model, the IoT nodes have to perform long-term channel detection and endure single-channel interference, which necessitates more sophisticated power control mechanisms [13]. The green initiatives are still in their infancy, and many theoretical and practical issues need to be thoroughly discussed; such analyses will boost the development of large-scale and hybrid IoT networks.

To the best of our knowledge, there is no design for a combined network of LoRa and ZigBee that addresses the energy efficiency issues in IoT devices. We take advantage of suitable distance and channel characteristics of both communications technologies to form a QoS-aware green IoT network. The design goal of this article is to develop a comprehensive design of a new type of green IoT network. The 
study contains node clustering, throughput optimization, energy self-management, and a field test. This network reduces the energy consumption of the IoT network while at the same time guaranteeing QoS. We believe that our new type of IoT node organization and control process could improve the performance of green-oriented IoT services and even change people's service use habits.

The remainder of this paper is organized as follows. Section II discusses the motivation of our design and introduces the organization of LoRa and ZigBee in a moderate-density IoT network. Afterwards, the implementation of multiple-hop data transmission is discussed. Section III gives a brief overview of the solution regarding the problem of organizing a large number of nodes, and a center node selection method is introduced for the overall energy efficiency. Based on that background, we further develop a multiple-hop scheduling algorithm to maximize the throughput of our designed low-rate long-distance IoT network. In Section IV, for general IoT nodes, we design an AI-based power control system for switching to sleep mode. In Section V, we describe a real-world deployment of the proposed design using Raspberry $\mathrm{Pi}$ and discuss the outlook for future network deployments. In Section VI, we conclude this paper by highlighting the green approaches.

\section{LoRA AND ZigBeE Ad Hoc NeTwORK}

Most of the unlicensed industrial, scientific, and medical frequency bands operated by LPWA use center frequencies of $2.4 \mathrm{GHz}, 868 / 915 \mathrm{MHz}$ (ZigBee), $433 \mathrm{MHz}$ (LPD433), and $169 \mathrm{MHz}$ (SRD). The first design of short-range IoT devices was limited to line-of-sight distance [14]. For example, ZigBee can maintain the speed of $250 \mathrm{kbit} / \mathrm{s}$, and Bluetooth can maintain the speed of $2 \mathrm{Mbit} / \mathrm{s}$ [15] [16], while the communication ranges are limited to 100 meters or even less [17]. Although receivers such as ZigBee have very high sensitivity [18], they cannot expand to cellular-like coverage ranges due to the distinct channel disturbance and poor penetration. With the exponential growth of the number of IoT devices, it is foreseeable that the topology of these devices will extend as well as that of cellular networks [19]. Due to the signaling overhead and inability to avoid multiple hops, the supported flow-level throughput is much lower than the theoretical transmission rate. The channel environment is also entirely different from that of a single-hop transmission. The actual bandwidth requirements are unsatisfied by a large-scale IoT network [20], even if bandwidth usage is composed of intermittent and sporadic data transmissions required by edge sensors. Additionally, packet loss and delay become problematic, and the situation could be even worse in an energy-hungry scenario.

A groundbreaking alternative solution is to use the unlicensed sub-gigahertz radio frequency band, widely known as LoRa [21]. Using the existing infrastructure of cellular networks, IoT devices can 


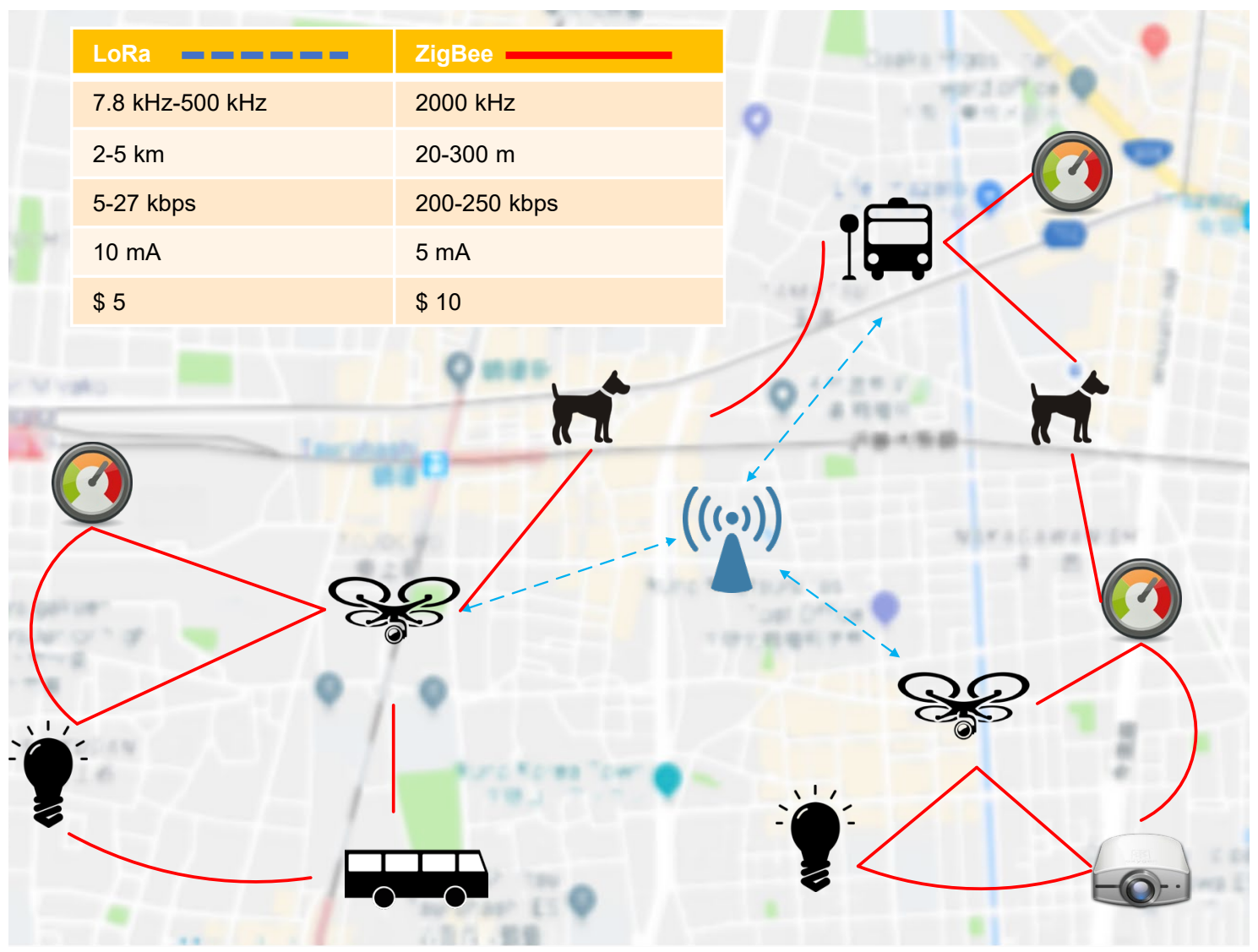

Fig. 2: Citywide topology of a hybrid network using LoRa and ZigBee.

connect with the base station using one-hop LoRa links [22], which have already been deployed by network operators in, e.g., France, the Netherlands, and Portugal. The advantages of these deployments are the remote connection ability and latency control. They help implement many interval-based services, such as street lights, temperature sensors, traffic control barriers, or other intelligent transportation systems. LoRa inherits the basic ideas of legacy cellular networks, where the gateway acts as a point of access for connecting to the IP-based core network [23]. However, the lack of resource allocation and device mobility awareness makes the topology less effective [24], and it is not particularity suitable for a large-scale and hybrid IoT deployment.

In summary, the existing solutions for large-scale hybrid IoT networks are limited by either the efficiency of single-level topology or the use of a very busy narrow frequency band. Hence, a three-layer topology for an LPWA-based hybrid network is introduced here. We design a hybrid transmission model and study the energy-saving measures for each IoT device to develop a QoS-aware green IoT network for the new era. 


\section{Overview of Network Organization}

As shown in Fig. 2, IoT nodes can form an ad hoc network through ZigBee or use LoRa that connects with the base stations (BSs) directly. In contrast to the traditional ZigBee topologies (e.g., star, tree or mesh topologies), some nodes are selected to relay collected information to the remote BSs; such relaying uses LoRa instead to guarantee that the transmission is uninterrupted and uses an exclusive channel. The other edge nodes are generally regarded as energy-hungry nodes. Thus, they must be activated from sleep mode first; subsequently, they transmit packets to relays. In contrast, relay nodes should listen to any incoming data from other IoT nodes or control information from the outside [25]. Some other nodes, which have greater mobility and larger capacity, are considered as flexible nodes and used to handle and store delay tolerance packets; such nodes subsequently send those packets to relays or BSs.

It is convenient that, on most occasions, both ZigBee and LoRa use the typical star topology. Both protocols are defined at the physical layer (PHY), using the unlicensed band (e.g., the mentioned band centered at $2.4 \mathrm{GHz}$ ), which makes the channel and subbands easy to distribute. The nodes with full function devices (FFDs) establish connections, set up routing and manage this network. They reduce the signal to environmental noise ratio using power control and account for the interference created by nearby Wi-Fi networks [26]. In addition, the nodes vary the length of spreading code to determine the variable data rates [27], which involves a trade-off between throughput, energy consumption, and robustness.

\section{Selectable Transmission Model}

The necessary condition for establishing a ZigBee and LoRa network is accessibility. As shown in Fig.3(a), nodes $A$ and $B$ are equipped with two types of protocols to relay messages between local nodes and connect to a remote BS. If other ZigBee embedded nodes want to communicate with the outside (e.g., node a communicates with node b), they will search the other nodes within the ZigBee range to use for relaying their data.

For each node, we use two queues to discuss various scenarios. As shown in Fig.3(b), the source node (denoted by S) creates two queues: a local queue, and a relay queue. When a local packet arrives, it is put at the end of the local queue. Node $S$ dispatches the packet at the head of a local queue, and the remaining packets advance in the queue. Then, the packet currently at the head of the queue becomes the next packet to be sent. In contrast, a packet received from one of the other nodes is kept in a relay queue if node $S$ is not the destination of that packet. If node $S$ is considered as the destination, there is a receive queue for node $S$ to store the packets expected to be received. The queue stores the sequence numbers 


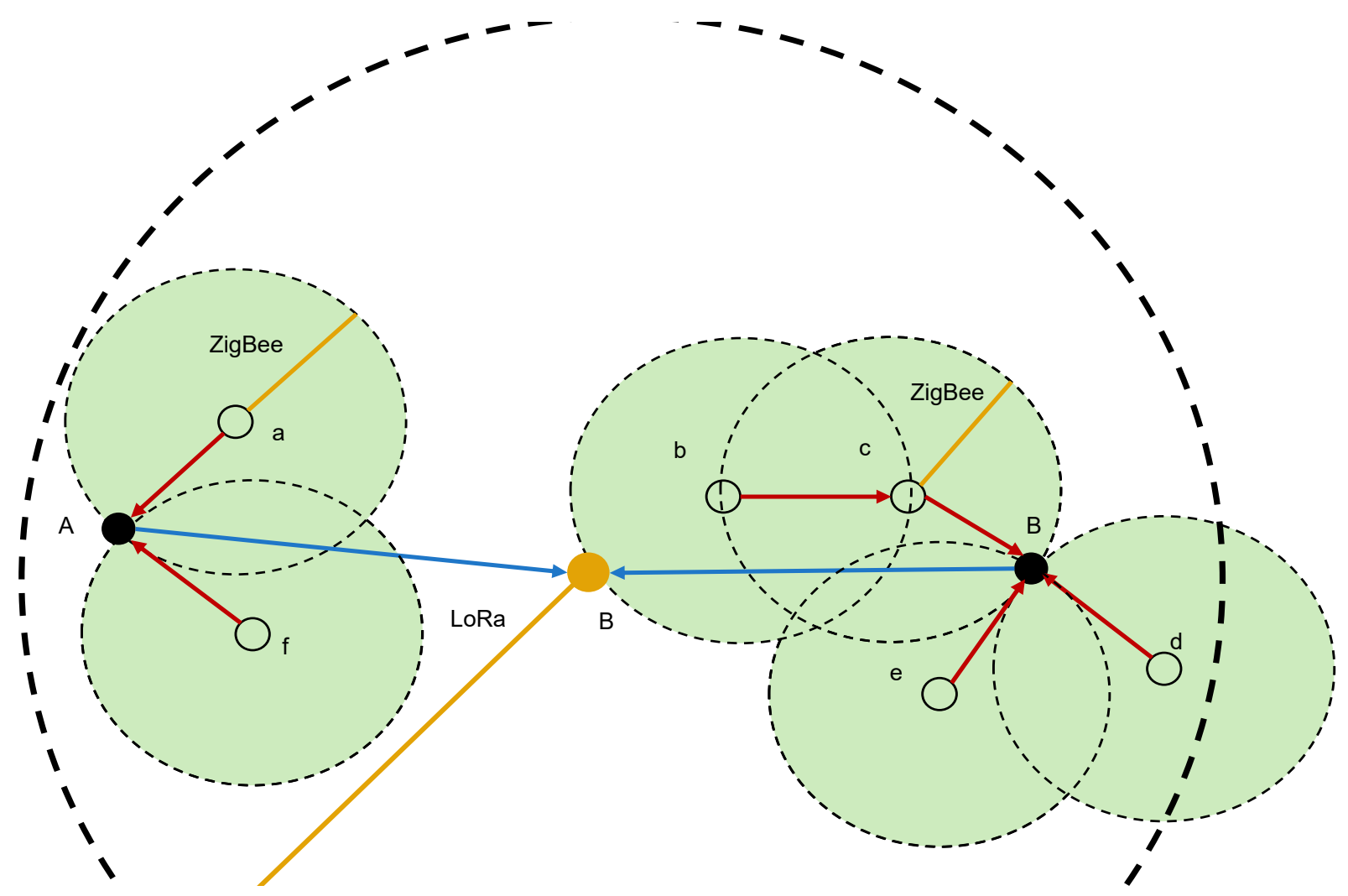

(a) Two different ways of message transmission depending on different protocols.

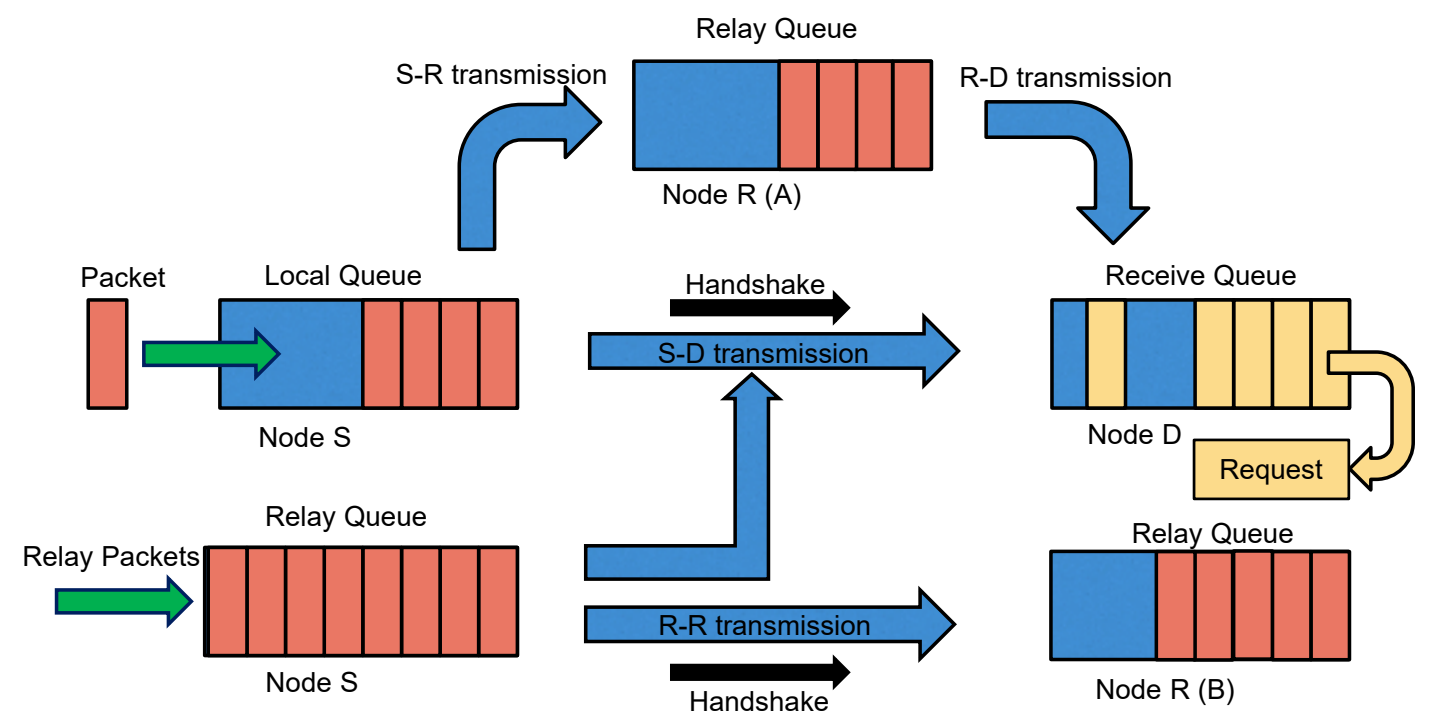

(b) Message relaying and forwarding process.

Fig. 3: The transmission and relay process of a hybrid green IoT network.

of packets that have not been received yet, and the corresponding packet's number will be removed after the packet arrives.

There are three types of transmission in the packet forwarding process: source to relay (S-R), relay to 
relay (R-R), and source to destination (S-D). The S-R transmission is designed for the start of multiple hop scheduling [28] and is used to share control information in the local area or collect monitored data at the sink node, using its ZigBee. A relay could refuse the transmission if that node was selfish enough (e.g., energy-hungry or experiencing a full relay queue). The R-R transmission is considered a bridge for hub nodes with sufficient energy; in this transmission mode, some redundant packets are deleted due to excessive forwarding and after a sufficiently long delay. The S-D and relay to destination (R-D) transmissions are designed to transmit packets through LoRa remotely to the BS or share data with local nodes through ZigBee. After a handshake, node $\mathrm{S}$ listens to the channel until it is empty and then transmits the packet to its destination.

\section{MULTIPLE HOP SCHEDULING IN AD HOC NETWORK}

\section{Automatic Center Node Selection}

In this network structure, nodes directly connected to the BS (e.g., the LoRa gateway) form an important bridge. They should be selected carefully, since the capacity of relaying between BS, and edge nodes corresponds to the actual coverage. Additionally, the battery and the hybrid transmission module are essential factors [29]. The traditional k-means or other mean-shift methods provide efficient ways of calculating the geometric centers of IoT nodes. However, due to the competition of WLAN channels in the local area and the indeterminate number of group members (various types of IoT devices), we need to design an algorithm that selects clusters that have nearby members and that are relatively remote from other potential clusters. Inspired by the efficient and low-complexity density clustering method in [30], we use the local density $p_{i}$ and the distance to a higher density node $\theta_{i}$ to distinguish the singular cluster from others. The local density $p_{i}$ for node $\mathrm{i}$ is defined as $p_{i}=\sum_{j} \exp \left(-\left(\frac{d_{i j}}{d_{c}}\right)^{2}\right)$, where $d_{c}$ is the cutoff distance. This equation represents the density of nodes in a proper communication range of node $i$. The reason is that the density only reflects the relative magnitude of various nodes, and it is a continuous function that has unequal values in the same set $S$. Thus, robustness is guaranteed. In addition, the distance to a higher density node $\theta_{i}$ is considered to be

$$
\theta_{i}= \begin{cases}\min \left(d_{i j}\right) \quad \text { if } \quad p_{j}>p_{i} \\ \max \left(d_{i j}\right) \quad \text { if } \quad p_{i} \text { is the highest, then } \mathrm{j} \text { is farthest node }\end{cases}
$$

Usually, this value is larger than the distance to the nearest node, and the potential center nodes are recognized as having higher values, since they are away from other stronger competitors. Then, we can 


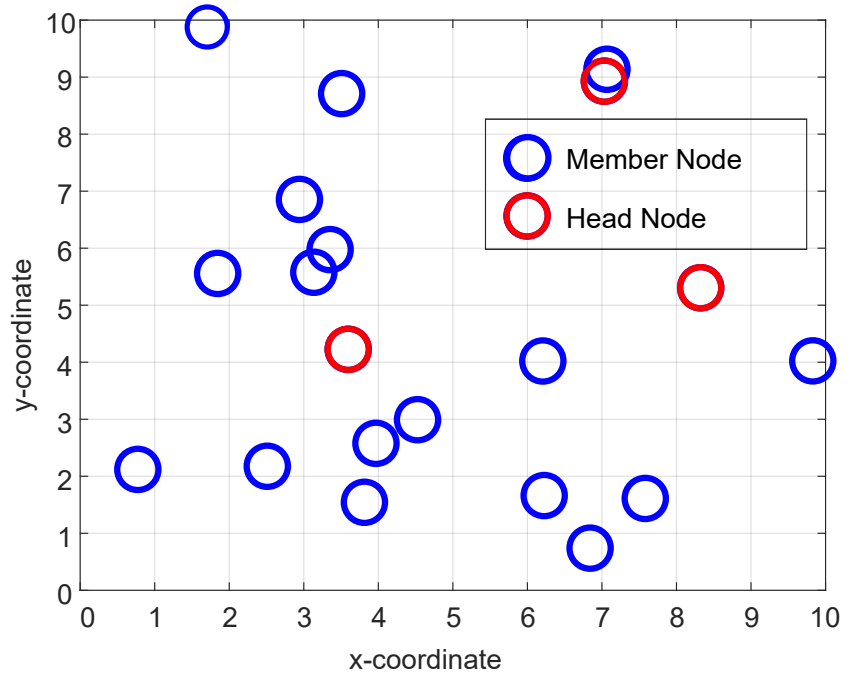

(a) Sample placement of nodes

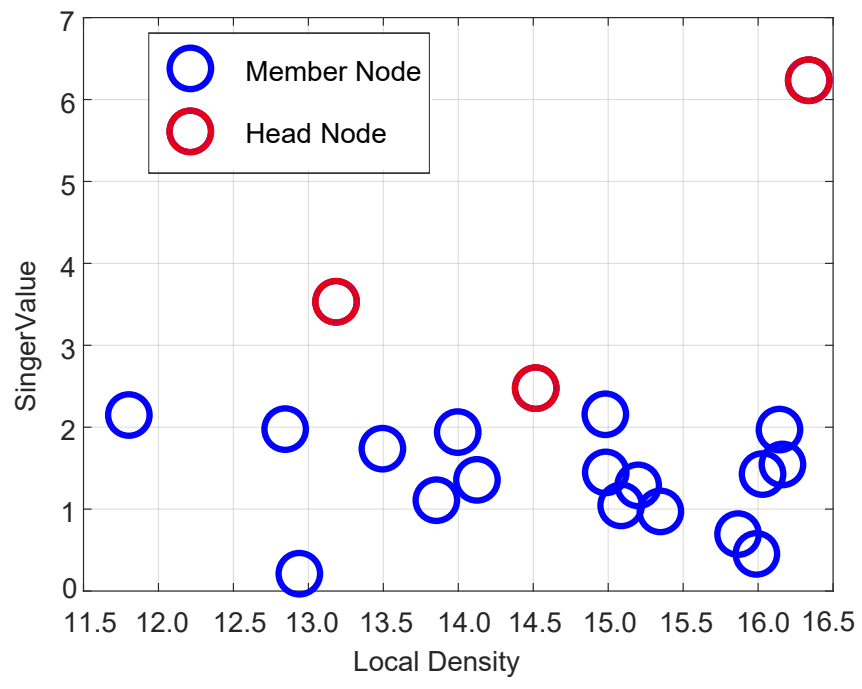

(b) Calculated singer value and local density

Fig. 4: Automatic cluster node selection based on placement.

express the outstanding cluster head clearly through the relaying node recommendation rate $R=\phi_{i} \theta_{i} p_{i}$ that is greater than threshold $R^{\prime}$, where $\phi_{i}$ is a binary number used to indicate whether the node is currently suitable as a relay due to reasons such as experiencing an energy shortfall or a malfunction. As shown in Fig. 4(a)(b), the method can intuitively identify the potential cluster head and determine the correct number of groups automatically.

\section{Multiple Hop Forwarding}

In a real-world communication environment, the capacity of the network is strongly related to the competition of links. Based on the mentioned protocols, the transmission link $i$ at time $t$ is stable if the following is satisfied for an arbitrary link $j:\left|T_{i}(t)-R_{i}(t)\right| \geq(1+\sigma)\left|T_{j}(t)-R_{i}(t)\right|$, where $\sigma$ is a predefined guard factor that depends on the protocol and guarantees that the interference problem is sufficiently small. $T_{i}$ and $R_{i}$ denote the positions of transmitter and receiver, respectively, and the absolute value sign that we use indicates the relative distance. If interference cannot be avoided, a selection of transmitters should be implemented. We use an equal opportunity mechanism to solve the competition problem. At each time interval, every node confirms whether there are active nodes nearby. If other nodes remain silent, it carries out the original plan. If other nearby nodes want to use this transmitting opportunity as well, they create a back-off counter with a number randomly selected from $[0, \mathrm{Ch}]$, where $C h$ is the number of channels. The value of $\mathrm{Ch}$ is based on the characteristics of the basic transmission protocol; e.g., we normally define 13 channels for Wi-Fi communication. Afterwards, the nodes listen to the channel until the counter runs 
out or until a handshake with a receiver. When a node's counter becomes 0 without any communication, the node claims itself as the only transmitter among nearby links. The probability of receiving a chance to transmit is the same for all nodes at the same level.

Next, we consider a standard multihop routing scheme with f-cast, where at most $f$ nodes in the transmission range are selected as the relays, and the packets sent by the source node are only stored at these $f$ nodes. For an arbitrary link $i$, the packets sent by the source node $S$ are labeled with a sequence number of packets $S N(P)$, and the destination correspondingly creates a required sequence number of packets $R N(D)$. The purpose of the sequence number is to guarantee that every packet is successfully transmitted to the destination in spite of transmission problems, such as packet loss or channel disturbance. The sequence numbers of received packets are removed from the list of remnant copies at the destination, as are the sequence numbers of packets that have been successfully transmitted outside by the source node.

Therefore, for the same sequence of two-hop packets, we can derive the probability $p_{1}$ that source $\mathrm{S}$ directly transmits the packet to destination and probability $p_{2}$ of the source transmitting it to a relay. Based on the channel competition mechanism mentioned before, we have

$$
\begin{aligned}
& p_{1}=\frac{1}{g^{2}}\left\{\frac{m-l / d}{l-l / d}\left(1-\left(\frac{l-l / d}{l}\right)^{l-l / d}+\frac{1}{l}\left(\frac{l-l / d}{n}\right)^{l-l / d}\right)\right\} \\
& p_{2}=\frac{1}{g^{2}}\left\{\frac{l-m}{l-l / d}\left(1-\left(\frac{l-l / d}{l}\right)^{l-l / d}-\left(\frac{l-m}{l}\right)^{l-l / d}\right)\right\}
\end{aligned}
$$

where $m=(2 d-l / d)^{2}, l$ indicates the length of a side of a square, and $d$ is the transmission range. Variable $g$ is the number of cluster heads that we automatically select through our center node selection algorithm. Then, if there are already $c(c \leq f)$ copies away from node $S$, we can derive the probability that the destination can receive, $p_{r}(c)=p_{1}+(c-1) /(2 l-4 l / d) \cdot p_{2}$.

Based on the above theoretical transmission model, we consider a new throughput optimization problem next.

\section{Optimized Multihop Scheduling}

In an IoT-formed MANET, where each packet is forwarded through multihop links with the limited transmission range $d$, the objective is to find a fixed number of relay nodes $f$ that maximizes the throughput per node. The meaning of this optimization is that, at peak traffic time, the capacity can always be maintained at the maximum level, if necessary. Furthermore, we introduce a new switching model for nodes capable of entering the sleep state to save energy in the next section; such a model turns off 


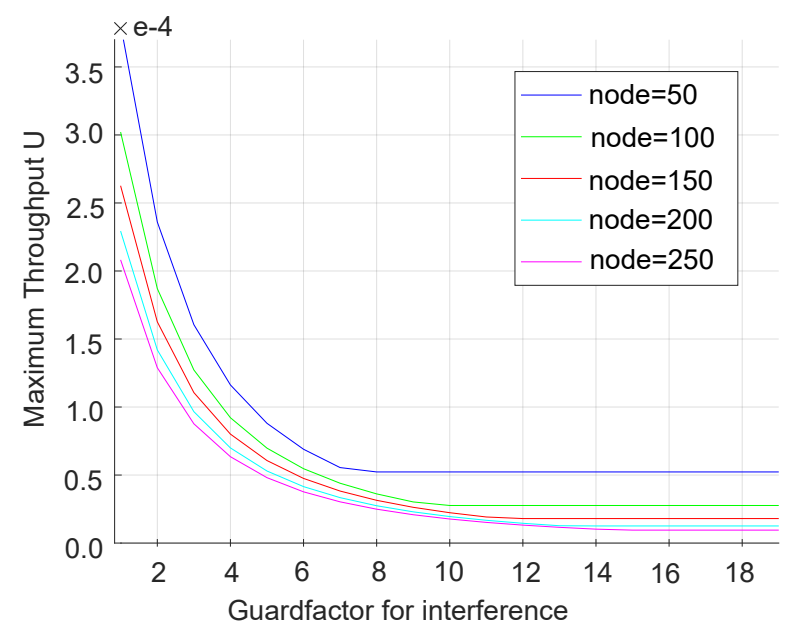

(a) Throughput changes by the predefined guard factor

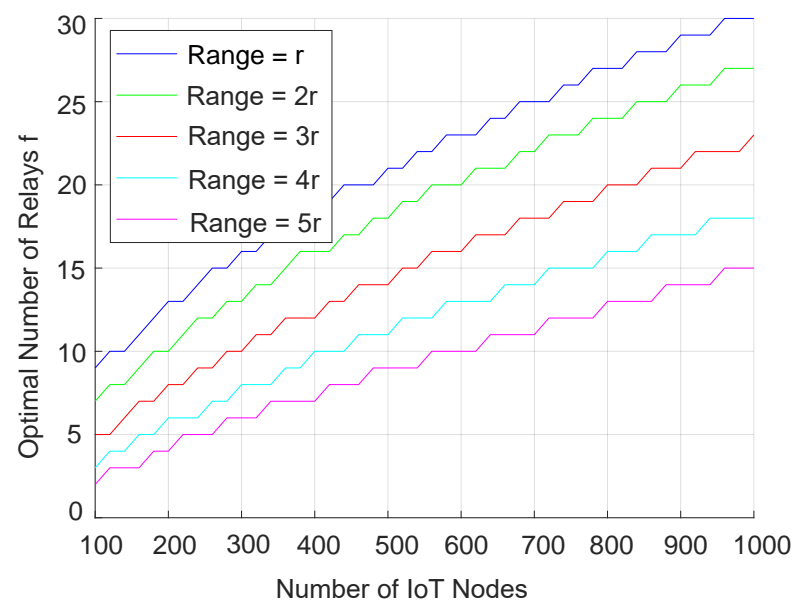

(b) Change in the optimal number of relays with network size

Fig. 5: Automatic throughput optimization.

unnecessary nodes regularly and minimizes the size of this network. Therefore, traffic of active nodes always remains high to finish every node's tasks, and the BS can wake up a part of nodes (e.g., more LoRa - BS links) to expand the network capacity.

Next, we optimize this variable-sized network. For a given transmission distance $d$, the maximum per-node throughput capacity $U$ is deduced as [31],

$$
\left.\left.U=\max \left\{1 /\left.E\left[X_{D}(f+1)\right]\right|_{f=f_{0}}\right], 1 /\left.E\left[X_{s}(1)\right]\right|_{f=f_{1}}\right]\right\}
$$

where $X_{s}(f)$ indicates the service time between when a packet starts to be delivered by node $S$ and when the distribution of copies ends, and $X_{D}(f)$ indicates the service time between when a packet starts to be requested by node $D$ and when it is received. In a classical star topology, variable $f_{0}$ indicates the maximum number of fixed nodes such that the expected service time $X_{s}$ is larger than that for only one relay, and it is defined as $f_{0}=\max \left\{f \mid E\left[X_{S}(1)\right] \leq E\left[X_{D}(f+1)\right]\right\}$. Variable $f_{1}$ indicates the minimum number of relaying nodes such that the expected service time $X_{D}$ is smaller than the distributing service time of using only one relay, defined as $f_{0}=\max \left\{f \mid E\left[X_{D}(f+1)\right] \leq E\left[X_{S}(1)\right]\right\}$. Based on the derivative process of the star topology mentioned in Eq. 3, we calculate the result in various settings. As shown in Fig. 5, it is easy to derive that the $f_{0}$ and $f_{1}$ in fact exist, since the expected service time $X_{S}(1)$ monotonically increases, while $X_{D}(f+1)$ monotonically decreases. Then, we can find fixed optimized settings $f_{0}$ and $f_{1}$ that together achieve the optimal throughput $U$. The optimized setting of $f$ is only influenced by the size of a network and has no relation with the time diversity. Therefore, the optimization algorithm is simplified to search for a specific $f$ corresponding to the changing size of the network. 


\section{SLeEP SWITCHING IN IoT DEVICES}

Previous studies show that the power control of base stations (BSs) and their next-level IoT devices can save approximately $60 \%$ to $80 \%$ of the overall energy consumption. Putting inactive nodes into the sleep mode is an efficient approach, as the traffic load is less than $10 \%$ of the peak value during $30 \%$ of the total time in one week [32]. The demand for IoT nodes, usually used in temperature sensors, lighting systems, and smart homes, is regular due to human life habits, which are strongly related with time and space. Transition into sleep mode can better balance the trade-off between the QoS requirement and energy consumption. The computational cost of data learning can be transferred from a single node to the BS. Generally, the learning of patterns for each IoT node is not a heavy burden on BSs [33] because the technologies of distributed computing and even fog computing can provide enough resources, and the goal of this learning is to 'guide' nodes as to how (or when) to wake up.

\section{Poisson Process-based Request Estimation}

Due to the limited storage and computational resources of energy-harvesting IoT devices, it is impossible to create a complicated schedule for each of them. Here, we design a traffic model based on an interrupted Poisson process (IPP) to describe the traffic requirement at each node. The goal is to search for a selfgenerating message transmission requirement threshold $N_{i}$ such that the transmission module would be active. The IPP has been proven to be influential in two-state traffic modeling, and it is widely used in various wireless traffic management technologies, such as 3GPP and LAN. We use the IPP model to perform a preliminary analysis of node behavior and, most importantly, to guarantee the delay performance and energy savings.

In a 24-hour service period, the periods of activity and inactivity are both exponentially distributed on

the timeline, with the average lengths of $r_{1}^{-1}$ and $r_{2}^{-1}$ [34], respectively. The active period begins when the node generates a packet and ends when the node finishes the packet's transmission. At each specific node, the transmission requirement rate $\lambda$ follows the Poisson process during the inactive period, and the average rate for one node is $\hat{\lambda}=\lambda r_{2} /\left(r_{1}+r_{2}\right)$. At each time, we assume that the node generates a packet of a usual size, and the transmission module remains inactive until $N$ packets are ready.

Our optimization goal is to make the sleep mode efficient; with an easily derived sleep probability $p_{s}$ based on the above $\lambda$, we obtain the expected total power consumption

$$
P=p_{s} P_{i n}+\left(1-p_{s}\right)\left(P_{\text {on }}+P_{t}\right)+P_{s} F
$$




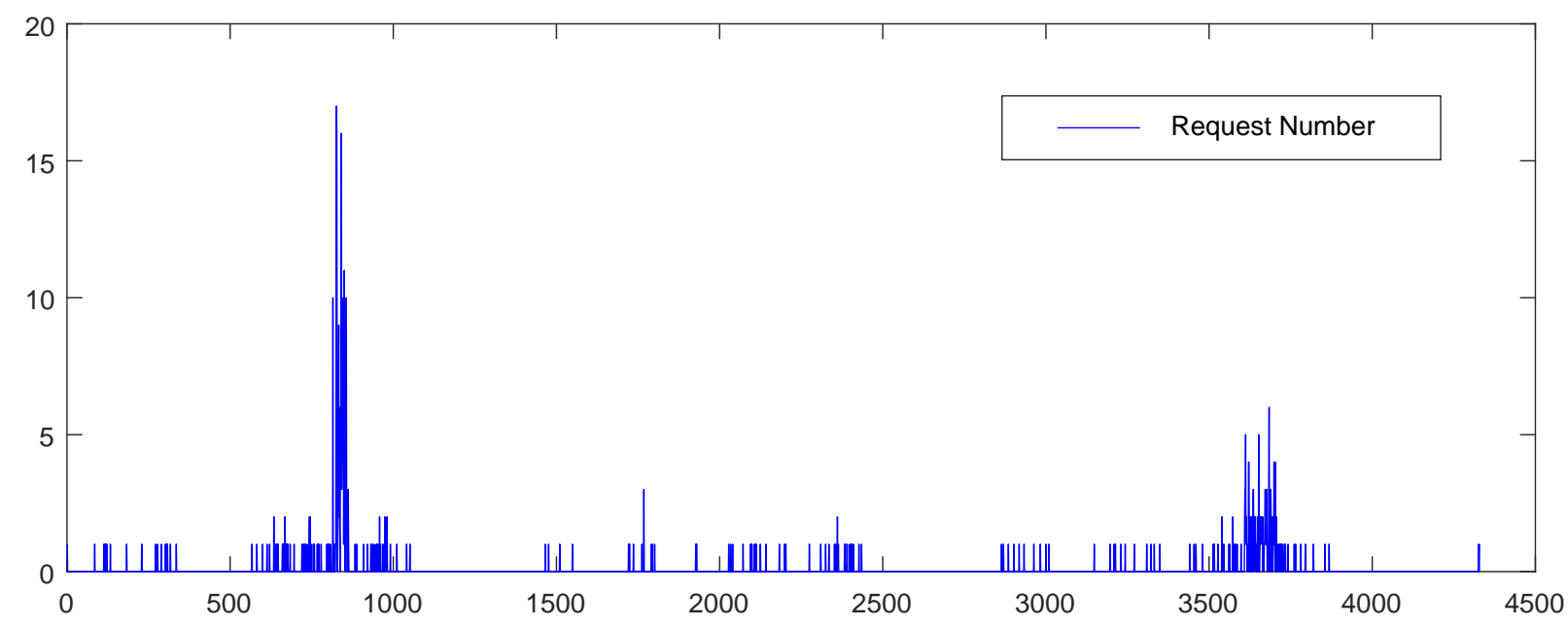

(a) Changes in the number of requests with the time stamp

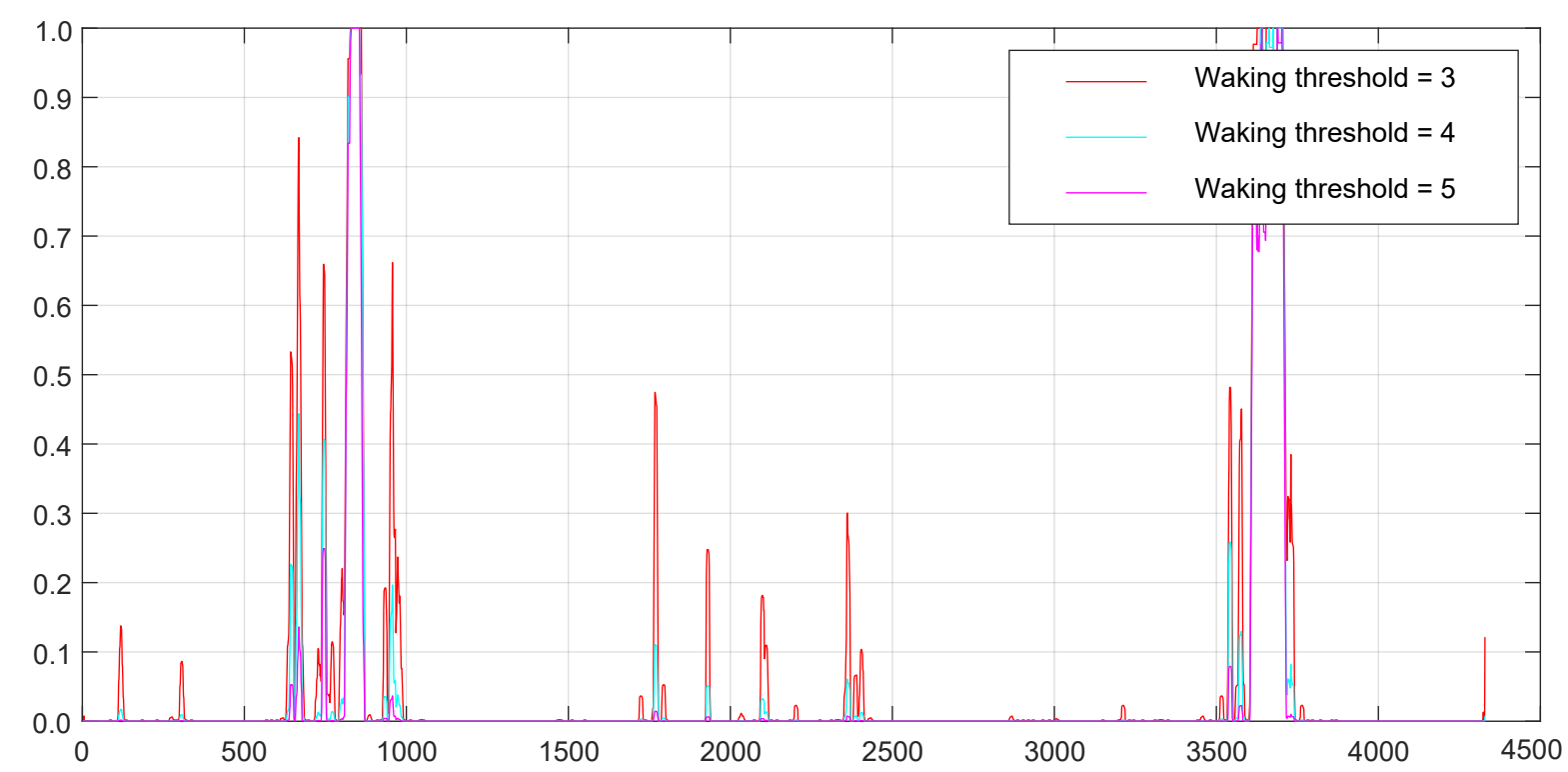

(b) Waking probability of a Poisson estimate based on the future trend's prediction

Fig. 6: Example of waking up nodes based on various waking thresholds.

According to the above equation, we can explore three energy-efficient approaches: increase the probability of the inactive mode with energy consumption $P_{i n}$, reduce the transmission power $P_{t}$ with the slope $\delta$ that indicates the power consumption depending on the load, or decrease the mode switching frequency $F$ associated with the switching cost $P_{s}$. Based on the IPP model, it is easy to prove that the packet threshold $N$ monotonically increases with $r_{2}$. Thus, a longer idle period corresponds to a larger packet redundancy threshold if energy consumption is optimized.

To determine the optimal set of packet redundancy $N$, we use the brute force search in small-scale data or 
directly deduce it using the approaches of previous studies. As shown in Fig. 6, each threshold corresponds to a different waking probability, which also refers to a different expected power consumption. The new results for the packet redundancy threshold $N$ and requirement rate $\lambda$ are indexed at the control module in BSs and sent to each corresponding node. When the service environment changes or an incident occurs, the algorithm uses the monotony of the optimal setting to calculate the packet redundancy efficiently. For example, in most of the cases, if the packet generating rate decreases, which leads to a larger probability of being in the inactive state, then we only need to search for a smaller redundancy threshold to minimize the power consumption (that is monotonically increasing with the active ratio).

\section{AI-controlled Power Switching System}

Using the data forwarding process, the BSs can always collect the activation records of each node, which could update the sleeping schedule based on such lagging data. The hysteretic data analysis is acceptable for timetable-based services (e.g., temperature sensors and signal lamps) but unsuitable for other human activity-oriented services (e.g., smart parking) and is even less appropriate in case of an emergency [35]. To overcome the considerable unreliability of analysis results obtained using historical data, we apply a new AI-controlled switching system to predict the future trend of the service request.

It is fortuitous that the environmental data collected by nearby IoT nodes are ultimately summarized

at the BSs (or a datacenter in an IP network), and these data are highly related with those collected by other IoT networks that interact with people. For instance, in the case of smart parking, drivers tend to use an indoor parking area rather than outdoor parking if a rainstorm is approaching. The above theory has also been proven in previous data mining studies and illustrates that regularly collected temporal and spatial data have a strong effect on the prediction result.

1) Big Data-based Prediction: As explained above, the data collected through the sensor network are very helpful in predicting the activity of IoT devices. Some critical data can effectively improve the accuracy of AI predictions if such data are properly collected and filtered. The selection of data can be based on empirical preprocessing, using the expertise of related services to select the relevant large environmental data of IoT devices and using the latter data as input to the predictive model. The weather data mentioned above, for example, have a more significant impact on outdoor service-oriented IoT networks. The selection of data can also be based on filtered results. One may extract some unusual samples of the device during the service process (e.g., when the device is overloaded or provides no service) and compare them to environmental data that also exhibit significant fluctuations at the same 
time or location. Using these large fluctuating data as training inputs improves the prediction of service demand with temporary fluctuating changes.

Training Models: The input data of the network model itself are based on the data collected by the diverse IoT networks. Predictions of a traditional fully connected network (e.g., an ANN) are inherently data fault-tolerant and robust to changes in the environment. Even if the data collected and filtered by hybrid IoT networks still have repetitive and negative correlations, a simple shallow neural network (or an RNN) with decision trees can effectively control the efficiency of energy-saving systems.

Model Deployment in IoT networks: Shallow neural networks have their advantages in deploying IoT devices. Such networks do not consume excessive amounts of computing resources, storing resources in and transferring resources from intermediate networks. Data preprocessing and network training are performed in the cloud [36]. Moreover, most of the same kinds of IoT devices can modeled by the same neural network. The central node in a cycle only needs to transfer the model data to the cluster head once, and then, the data are distributed to each edge device. This process does not take up too many valuable LPWAN resources, and nodes with mobile capabilities [37] can form new ZigBee links everywhere to effectively share spectrum resources.

2) Reinforcement Learning-based Predictions: Another kind of prediction that can be obtained based purely on the traffic control information of the network itself is the reinforcement learning model based on the hidden Markov chain. The advantage of this model is that it does not require a large quantity of environmental data as input and is filtered for storage.

The sample that we use is the record of service requirements and device control across the network, that is, self-optimization traffic control based on the network itself. Its advantages are being instantaneous, having a low initial demand, and being very predictable for sudden service increases and decreases. However, the predictive ability of long-term cyclical changes is weak, and a reasonable scale of learning needs to be preset to correct the adverse effects of reinforcement learning.

A hidden Markov chain (HMM) can effectively recover the history of the entire decision-making process based on the service appearances [38]. We only need to know the status $\mathrm{S}$ of network nodes, as well as the corresponding action A. This policy $\pi$, on the other hand, can use the mentioned IPP model as an initial value. The optimal policy is calculated by setting up an evaluation parameter Q (including the transmission overhead, the switching overhead, the reward for demand satisfaction, etc.). Afterwards, the learning formula

$$
Q(s, a)=Q(s, a)+\alpha\left(r(s, a)+\gamma \max _{a^{\prime}} Q\left(s^{\prime}, a^{\prime}\right)-Q(s, a)\right)
$$


can be used to influence the power control policy. Ultimately, a policy $\pi$ corresponding to the different values of the state matrix $S$ will be used as the solution for the power control system to maximize energy savings.

When the cloud collection of control data is large enough, we can even use deep reinforcement learning to train in the cloud; in the memory replay approach, a random selection of some historical control data is added to the recent reinforcement learning. The data are mixed into the input sample (as a mini-batch), thus balancing the impact of recent events on the overall network; the network clone technique involves setting up a parallel space for the predictive network Q'. Compared to learning after each round of data updating, learning and testing are completed only after the collection of M rounds of data, thus maximizing stability.

Using the trend prediction, the BSs can adjust the sleeping strategy over time, even if facing sudden changes. In general, the optimization of throughput or power only concerns the size of each message, and the value of content has not been thoroughly investigated. Because of the diversity of IoT nodes, the existence of various types of messages makes the optimization task more difficult. However, if we could effectively use the inner connections between these messages' content, our network management would be even more accurate.

\section{EXPERIMENT AND COMPARISONS}

\section{A. Implementation}

First, we perform the field test of our LoRa and ZigBee ad hoc network. Installing a LoRa gateway on top of a building allows all the nodes embedding LoRa modules to access the core network through that gateway. As shown in Fig. 7, the installation includes the integration of Raspberry Pi with a gateway module, connection with a database and registration with an online service. For an individual IoT node, the collected data can be transmitted to a remote antenna through either the LoRa module or the ZigBee module. At present, the installation has been completed, and the resulting network successfully delivers packets within ten kilometers.

\section{B. Network Flow Control}

Traffic flow control of the network is divided into two different dimensions. In practice, the power control of communication modules in the network is mainly divided into two steps: the establishment of an active node connection diagram and the network traffic flow control. When a new time frame 


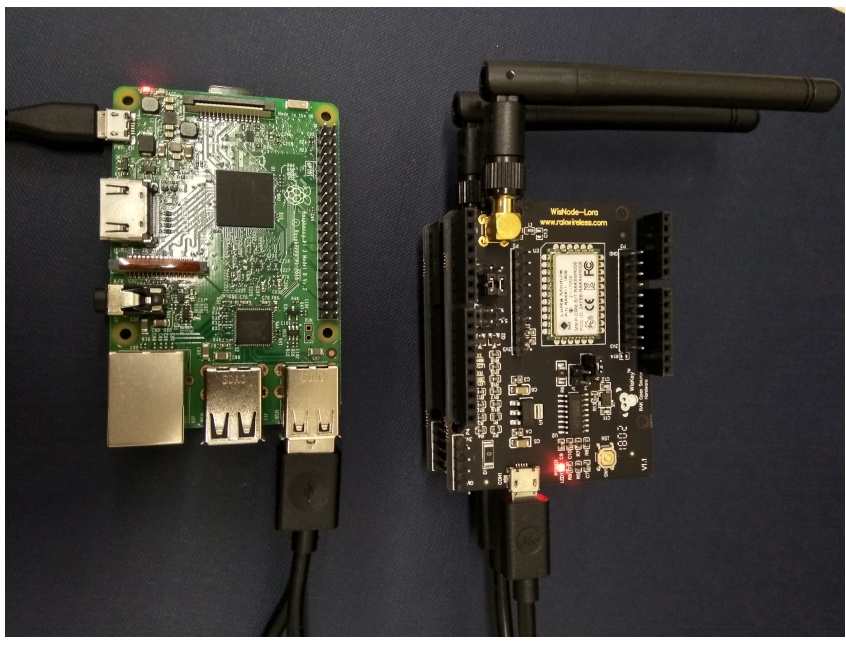

(a) Integration of Raspberry Pi with a gateway module

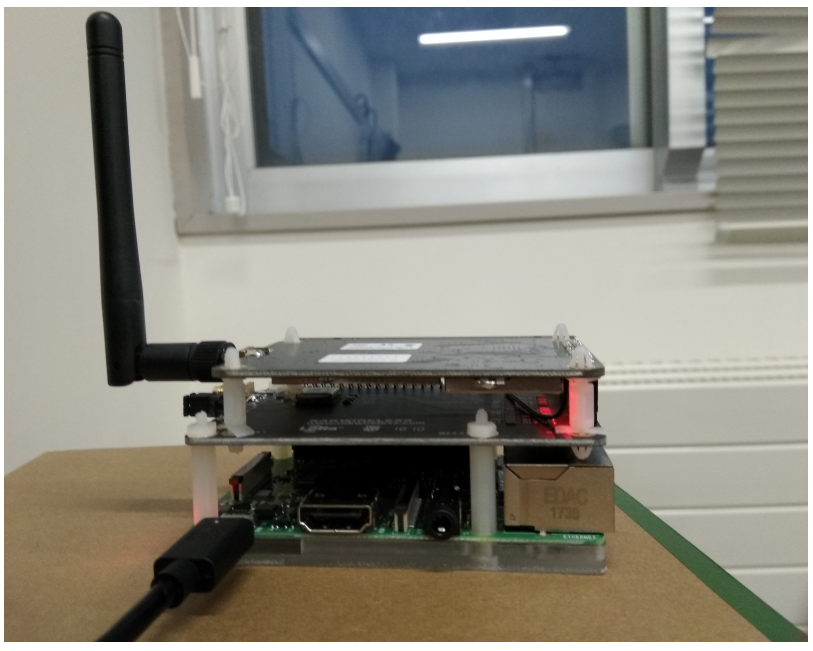

(b) User-side transmission module connecting with an IoT device

Fig. 7: Deployment of LoRa and ZigBee transmission module.

starts, each center node and the gateway are aware of the switch conditions for each edge node. In other words, the future control scheme of the previous power control system is also stored at these intermediate nodes that can form a network similar to that shown in Fig. 8(a). After establishing the temporary active node connection diagram, we can select a traffic control policy for data flows. According to the existing literature, the use of relay priorities that maximize hop number first and destination closeness first can optimize the overall network response delay and the total throughput, respectively [39].

Next, we continue and finish the optimization of the network settings. To understand the range setting clearly, we use the deduction process mentioned before, and the calculated results are shown in Fig. 8(b). The size of a star topology network varies from 100 to 400, and the maximum throughput varies from 0.0014 to 0.0052 per packet/slot. Five power levels are described that correspond to various communication ranges ( $\mathrm{r}=10$ meters in our simulation). At first, the longer transmission model performs better due to the low density of nodes, and it consumes more power to obtain a higher throughput. However, with increasing node density, the influence of different ranges becomes low, and the throughput at each range decreases significantly.

\section{Green Effect in IoT}

In Fig. 9(a), we show the calculated total energy consumption per hour. The blue bar corresponds to being constantly active, while bars labeled PeakA and LZAI represent being active during peak hours (regularly activated) and our proposed AI-controlled LZ system, respectively. Based on our observations, $15 \%$ to $25 \%$ of nodes are selected as the head nodes to transmit messages using LoRa, while the other 


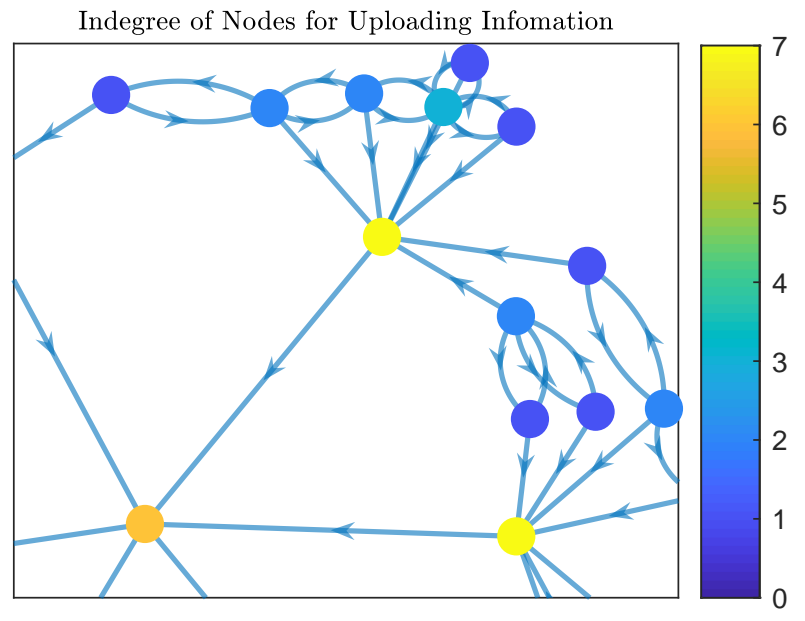

(a) Star-type topological structure formed in each time slot.

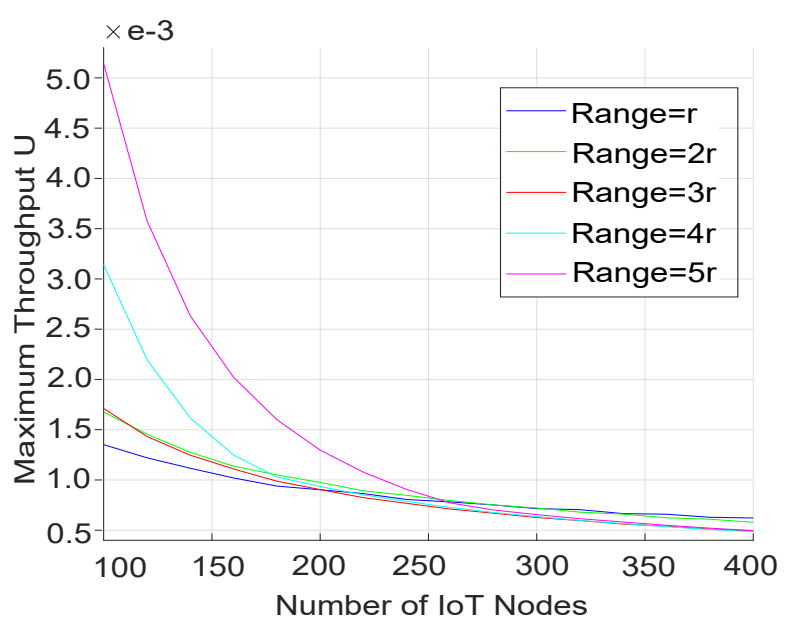

(b) Changes in the overall maximum throughput for various ranges.

Fig. 8: Network structure and throughput in the experiment.

ZigBee nodes save approximately one-half of the energy used if the nodes remain active all the time. However, due to the higher density of nodes, traffic congestion forces the nodes to spend more time monitoring the channel, which consumes more energy than expected.

In Fig. 9(b), we simulate and study the impact of various communication module configurations on the power-saving performance of the network. For such configurations, the ratio of the number of LoRa nodes to that of ZigBee nodes varies from $1 / 3$ to $1 / 6$. The number of nodes is approximately 400 . The cumulative results show that our LZAI strategy can play a significant role in saving energy. In the case of a larger number of LoRa node modules, the use of multiple communication modules in nodes will increase energy consumption. Moreover, when fewer selectable nodes are available for upward communication, Zigbee node trends to upload the data to the cluster node and switch off itself, which could further reduce energy consumption. It may increase the overall transmission latency. However, our network optimization architecture proposed before is to alleviate this adverse effect.

\section{CONCLUSION}

In this paper, we conduct an all-around solution for new green LoRa and Zigbee IoT ad hoc network, which realizes the self-regulation of composition, size minimization, and throughput optimization. At first, we propose a unique initialization mechanism that is automatically scheduling node clustering and throughput optimization. Then, a self-energy management method is conducted to optimize the sleep switching in each device, which through an AI-controlled service usage habit prediction method to learn 


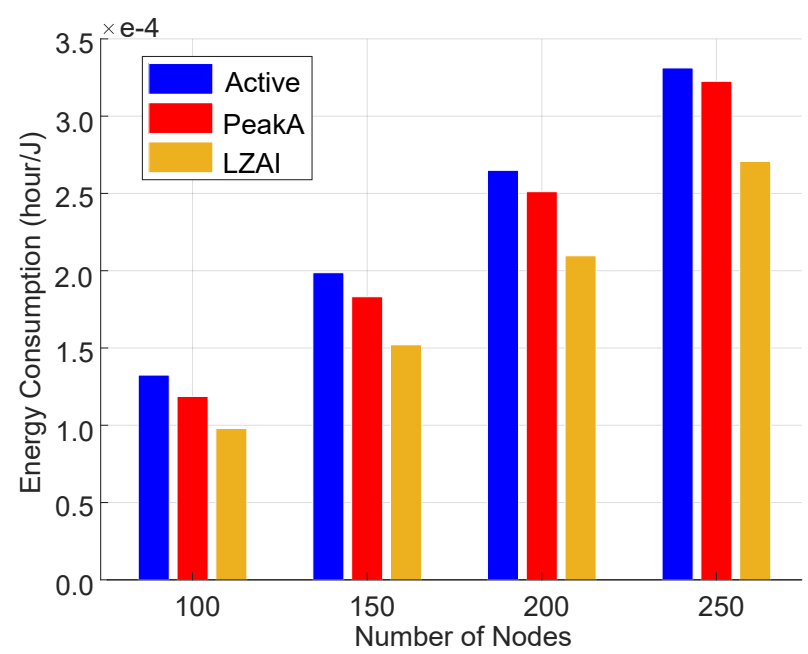

(a) Energy consumption using different strategies.

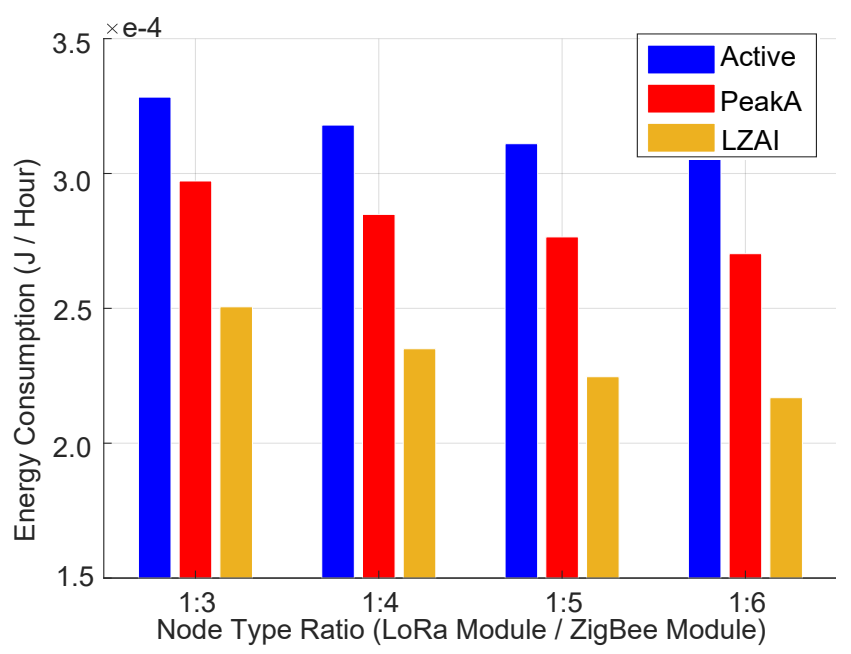

(b) Energy consumption under different setting.

Fig. 9: Large-scale comparison of LoRa and ZigBee IoT networks.

the future requirement trend. Different from other control systems with dynamic scheduling, our method provides a unique scheduling setting to approximate the optimal one, which significantly lightens the burden of storage, computation cost, and power. We believe our new design could improve the performance of real deployment IoT services, and even changes humans lifestyle habits through the efforts of our energy savings.

\section{ACKNOWLEDGEMENT}

This work is partially supported by JSPS KAKENHI Grant Number JP16K00117, and KDDI Foundation.

\section{REFERENCES}

[1] R. Arshad, S. Zahoor, M. A. Shah, A. Wahid, and H. Yu, "Green IoT: An investigation on energy saving practices for 2020 and beyond," IEEE Access, vol. 5, pp. 15667-15681, Jul 2017.

[2] A. Parsa, T. A. Najafabadi, and F. R. Salmasi, "Implementation of smart optimal and automatic control of electrical home appliances (IoT)," in Proc. of 2017 Smart Grid Conf. (SGC), pp. 1-6, Dec 2017.

[3] T. Yang, C. Yang, and T. Sung, "An intelligent energy management scheme with monitoring and scheduling approach for IoT applications in smart home," in Proc. of 3rd Int. Conf. on Robot, Vision and Signal Processing (RVSP), pp. 216-219, Nov 2015.

[4] M. A. Desima, P. Ramli, D. F. Ramdani, and S. Rahman, "Alarm system to detect the location of IoT-based public vehicle accidents," in Proc. of 2017 Int. Conf. on Computing, Engineering, and Design (ICCED), pp. 1-5, Nov 2017.

[5] U. Raza, P. Kulkarni, and M. Sooriyabandara, "Low power wide area networks: an overview," IEEE Commun. Surveys Tuts., vol. 19, pp. 855-873, Jan 2017. 
[6] A. N. Babadi, S. Nouri, and S. Khalaj, "Challenges and opportunities of the integration of IoT and smart grid in iran transmission power system," in Proc. of 2017 Smart Grid Conference (SGC), pp. 1-6, Dec 2017.

[7] R. Haight, W. Haensch, and D. Friedman, “Solar-powering the Internet of Things,” Science, vol. 353, pp. 124-125, Jul 2016.

[8] J. Liu, M. Sheng, Y. Xu, J. Li, and X. Jiang, “On throughput capacity for a class of buffer-limited MANETs," Ad Hoc Networks, vol. 37, pp. 354 - 367, Feb 2016.

[9] S. Sen, J. Koo, and S. Bagchi, "Trifecta: Security, energy efficiency, and communication capacity comparison for wireless IoT devices," IEEE Internet Comput., vol. 22, pp. 74-81, Jan 2018.

[10] J. Chen, K. Hu, Q. Wang, Y. Sun, Z. Shi, and S. He, “Narrowband Internet of Things: Implementations and applications,” IEEE Internet Things J., vol. 4, pp. 2309-2314, Dec 2017.

[11] P. Ran, M. h. Sun, and Y. m. Zou, "Zigbee routing selection strategy based on data services and energy-balanced ZigBee routing," in Proc. of 2006 IEEE Asia-Pacific Conf. on Services Comput. (APSCC), pp. 400-404, Dec 2006.

[12] D. Li, M. Dong, Y. Yuan, J. Chen, K. Ota, and Y. Tang, "Seer-MCache: A prefetchable memory object caching system for IoT real-time data processing," IEEE Internet Things J., vol. 5, pp. 3648-3660, Oct 2018.

[13] M. Tao, K. Ota, M. Dong, and Z. Qian, "Access Auth: Capacity-aware security access authentication in federated-IoT-enabled V2G networks," J. Parallel Distrib. Comput., vol. 118, pp. 107 - 117, Aug 2018.

[14] J. Wu, L. Zou, L. Zhao, A. Al-Dubai, L. M. Mackenzie, and G. Min, "A Multi-UAV clustering strategy for reducing insecure communication range," Comput. Networks, Apr 2019.

[15] P. Yi, A. Iwayemi, and C. Zhou, "Developing ZigBee deployment guideline under wifi interference for smart grid applications," IEEE Trans. Smart Grid, vol. 2, pp. 110-120, Mar 2011.

[16] P. Baronti, P. Pillai, V. W. Chook, S. Chessa, A. Gotta, and Y. F. Hu, "Wireless sensor networks: A survey on the state of the art and the 802.15.4 and ZigBee standards," Comput. Commun., vol. 30, pp. 1655 - 1695, May 2007.

[17] M. Siekkinen, M. Hiienkari, J. K. Nurminen, and J. Nieminen, "How low energy is bluetooth low energy? comparative measurements with ZigBee/802.15.4," in Proc. of 2012 IEEE Wireless Commun. and Netw. Conf. Workshops (WCNCW), pp. 232-237, Apr 2012.

[18] G. Pekhteryev, Z. Sahinoglu, P. Orlik, and G. Bhatti, "Image transmission over IEEE 802.15.4 and ZigBee networks," in Proc. of 2005 IEEE Int. Symposium on Circuits and Systems. (ISCAS), pp. 3539-3542 Vol. 4, May 2005.

[19] M. Tao, K. Ota, and M. Dong, "Locating compromised data sources in IoT-Enabled smart cities: A great-alternative-region-based approach,” IEEE Trans. Ind. Informat., vol. 14, pp. 2579-2587, Jun 2018.

[20] G. Guan, K. Fu, Z. Cheng, Y. Gao, and W. Dong, "Rapid development of IoT applications with TinyLink," in Proc. of 2017 IEEE Conf. on Comput. Commun. Workshops (INFOCOM WKSHPS), pp. 956-957, May 2017.

[21] A. Hoeller, R. D. Souza, O. L. Alcaraz, H. Alves, M. de Noronha Neto, and G. Brante, "Exploiting time diversity of LoRa networks through optimum message replication,” in Proc. of 15th Int. Symposium on Wireless Commun. Syst. (ISWCS), pp. 1-5, Aug 2018.

[22] K. Ke, Q. Liang, G. Zeng, J. Lin, and H. Lee, "Demo abstract: A LoRa wireless mesh networking module for campus-scale monitoring," in Proc. of 16th ACM/IEEE Int. Conf. on Information Processing in Sensor Networks (IPSN), pp. 259-260, Apr 2017.

[23] M. Centenaro, L. Vangelista, A. Zanella, and M. Zorzi, "Long-range communications in unlicensed bands: The rising stars in the IoT and smart city scenarios," IEEE Wireless Commun., vol. 23, pp. 60-67, Oct 2016.

[24] O. Novo, "Blockchain meets IoT: An architecture for scalable access management in IoT," IEEE Internet Things J., vol. 5, pp. 11841195, Apr 2018.

[25] L. Zhao, A. Al-Dubai, X. Li, G. Chen, and G. Min, "A new efficient cross-layer relay node selection model for wireless community mesh networks," Comput. Electr. Eng., vol. 61, pp. 361-372, Jul 2017.

[26] S. P. Mohanty, U. Choppali, and E. Kougianos, "Everything you wanted to know about smart cities: The Internet of things is the backbone," IEEE Consum. Electr. Mag., vol. 5, pp. 60-70, Jul 2016. 
[27] M. Tao, K. Ota, and M. Dong, "Ontology-based data semantic management and application in IoT- and cloud-enabled smart homes," Future Gener. Comp. Syst., vol. 76, pp. 528 - 539, 2017.

[28] W. Liu, K. Nakauchi, and Y. Shoji, "A neighbor-based probabilistic broadcast protocol for data dissemination in mobile IoT networks," IEEE Access, vol. 6, pp. 12260-12268, Mar 2018.

[29] C. Xu, L. Yang, and P. Zhang, "Practical backscatter communication systems for battery-free Internet of Things: A tutorial and survey of recent research,” IEEE Signal Process. Mag., vol. 35, pp. 16-27, Sep 2018.

[30] R. Alex and L. Alessandro, "Clustering by fast search and find of density peaks," Science, vol. 344, pp. 1492-1496, Dec 2014.

[31] J. Liu, X. Jiang, H. Nishiyama, and N. Kato, "Exact throughput capacity under power control in mobile ad hoc networks," in Proc. of 2012 IEEE Conf. on Comput. Commun. (INFOCOM), pp. 1-9, Mar 2012.

[32] X. Luo, J. Liu, D. Zhang, and X. Chang, "A large-scale web QoS prediction scheme for the industrial internet of things based on a kernel machine learning algorithm," Comput. Networks, vol. 101, pp. 81-89, Jun 2016.

[33] H. Li, K. Ota, and M. Dong, "Learning IoT in edge: Deep learning for the Internet of Things with edge computing," IEEE Netw., vol. 32, pp. 96-101, Jan 2018.

[34] J. Wu, Y. Bao, G. Miao, S. Zhou, and Z. Niu, "Base-Station sleeping control and power matching for energy delay tradeoffs with bursty traffic,” IEEE Trans. Veh. Technol., vol. 65, pp. 3657-3675, May 2016.

[35] L. Li, K. Ota, and M. Dong, "When weather matters: IoT-based electrical load forecasting for smart grid," IEEE Commun. Mag., vol. 55, pp. 46-51, Oct 2017.

[36] A. Botta, W. de Donato, V. Persico, and A. Pescapé, "Integration of cloud computing and Internet of Things: A survey," Future Gener. Comp. Syst., vol. 56, pp. 684-700, Mar 2016.

[37] C. Gong, M. Li, L. Zhao, Z. Guo, and G. Han, "Homomorphic evaluation of the integer arithmetic operations for mobile edge computing," Wireless Commun. and Mob. Com., vol. 2018, pp. 1-13, Nov 2018.

[38] W. Wei, X. Fan, H. Song, X. Fan, and J. Yang, "Imperfect information dynamic Stackelberg game based resource allocation using hidden markov for cloud computing," IEEE Trans. on Services Computing, vol. 11, pp. 78-89, Jan 2018.

[39] M. V. Moreno, J. Santa, M. A. Zamora, and A. F. Skarmeta, "A holistic IoT-based management platform for smart environments," in Proc. of 2014 IEEE Int. Conf. on Commun. (ICC), pp. 3823-3828, Jun 2014. 